Sergey I. Agafonov(D)

\title{
Note on generic singularities of planar flat 3-webs
}

Received: 9 January 2016 / Accepted: 8 December 2016

Published online: 21 December 2016

Abstract. We propose a definition of genericity for singular flat planar 3-webs formed by integral curves of implicit ODEs and give a classification of generic singularities of such webs.

\section{Introduction}

A planar $d$-web is formed by $d$ foliations in the plane. At each point of the plane, we have $d$ leaves passing through the point, one from each foliation of the web. A point is called regular if any two of these $d$ leaves are transverse. Consider the pseudogroup of local diffeomorphisms of the plane and the corresponding equivalence relation on the set of $d$-web germs. Any two planar 2-web germs are equivalent whenever the base points are regular. This is not true for 3-web germs (see [10]). There is a local invariant, which has, in fact, a topological nature. The differentialgeometric counterpart of this invariant is the so-called Blaschke curvature.

Definition 1. 3-web is flat (or hexagonal) if its germ at any regular point is equivalent to the web formed by three families of parallel lines.

A 3-web is flat if and only if the Blaschke curvature vanishes identically (see, for instance [10]). This curvature is a scalar 2-form, therefore any general classification of 3-webs with respect to local diffeomorphisms will necessarily have functional moduli. Namely, such a classification will inevitably involve arbitrary functions of two variables.

By the above definition, any two flat 3-web germs are equivalent provided that the base points are regular. Hence the "personality" of a flat 3 -web is encoded in its singularities.

Web structure is ubiquitous in mathematics and its applications, the Blaschke curvature often being the obstacle to obtaining a "reasonable" classification. Therefore flat 3-webs play a distinguished role.

For example, hexagonal 3-webs have a 3-dimensional local symmetry algebra at regular points, while a generic 3 -web does not admit any infinitesimal symmetry

S. I. Agafonov ( $\varangle$ ): Departamento de Matemática, UNESP-Universidade Estadual Paulista, São José do Rio Preto, Brazil. e-mail: agafonov@ibilce.unesp.br

Mathematics Subject Classification: Primary 53A60; Secondary 34M35 
(see [11]). (By infinitesimal symmetry of the web we understand a vector field whose local flow preserves the web.)

It was also observed that characteristic 3 -webs of integrable equations, playing important role in mathematical and theoretical physics, are flat (see, for instance, $[6,17,18,21])$.

The singularities of planar 2-webs, defined by solutions of implicit ODEs, quadratic in the derivative, are well understood (see [7,14,26]), whereas singular implicit ODEs, polynomial in derivatives of degree $d \geq 3$ bring more difficulties, the main obstacle being the nontrivial web structure on its solutions (see $[13,14$, 20,23,25]).

Let us review some known results on classification of flat 3-webs. One can show that a general classification of singular flat 3-webs will also have functional moduli, now the "moduli" being arbitrary functions of one variable (see, for example, the discussion in [1]). To get a reasonable classification one has to restrict the class of admissible singularities by imposing some meaningful conditions.

A sufficiently general class of singular 3-web germs can be described by binary forms:

$$
K_{3}(x, y) d y^{3}+K_{2}(x, y) d y^{2} d x+K_{1}(x, y) d y d x^{2}+K_{0}(x, y) d x^{3}=0 .
$$

Dividing the above form by $d x^{3}$ one gets an implicit ODE, cubic in $p=\frac{d y}{d x}$ :

$$
K_{3}(x, y) p^{3}+K_{2}(x, y) p^{2}+K_{1}(x, y) p+K_{0}(x, y)=0 .
$$

Equation (1) defines a (possibly singular) surface $M$ in 3-dimesional contact space $\mathbb{R}^{2} \times \mathbb{P}^{1}(\mathbb{R})$. It is immediate that if a point $(x, y)$ is not regular then the projection $\pi: M \rightarrow \mathbb{R}^{2},(x, y, p) \mapsto(x, y)$ is not a local diffeomorphism at least at one point $m$ in the fiber $\pi^{-1}(x, y)$.

Define the criminant $C$ of implicit ODE (1) as the set of points on $M$, where the projection $\pi$ fails to be a local diffeomorphism, and the discriminant $\Delta$ of Eq. (1) (or the apparent contour of the surface $M$ ) as the image of the criminant under the projection: $\Delta=\pi(C)$.

Under some natural conditions of regularity for the surface $M$ and for the criminant $C$, implying the smoothness of $M$ and $C$, (see Eq. (8) in the next section), the following normal forms were obtained in [1]:
1) $p^{3}+p x-y=0$
2) $p^{3}+2 x p+y=0$,
3) $p^{2}=x$,
4) $p^{2}=y$.

Remark. For the quadratic ODEs, the third root is $\infty$. The cubic normal forms were conjectured in $[24,25]$.

Another natural restriction on the singularity is the existence of at least one infinitesimal symmetry at the singular point. It turns out that at a singular point generators of a symmetry algebra can become infinite or multi-valued, thus this restriction is not trivial (see [2]). We call a web homogeneous if it is invariant with respect to the flow of Euler vector field

$$
E=c_{1} x \frac{\partial}{\partial x}+c_{2} y \frac{\partial}{\partial y}, \quad c_{i}=\text { const. }
$$


Singular homogeneous 3-webs were classified in [3]. To give the reader some idea about the functions emerging in this classification, we present here some normal forms:

$$
\begin{aligned}
& p^{3}+y^{2} p=\frac{2}{\sqrt{27}} y^{3} \tan (2 \sqrt{3} x) \\
& p^{3}+4 x\left(y-\frac{4}{9} x^{3}\right) p+y^{2}+\frac{64}{81} x^{6}-\frac{32}{9} y x^{3}=0, \\
& p^{3}+y^{3} p=y^{\frac{9}{2}} U\left(x y^{\frac{1}{2}}\right),
\end{aligned}
$$

where $U$ is expressed in terms of the Legendre functions $P_{v}^{\mu}(z), Q_{v}^{\mu}(z)$. Note that all the forms (2) also are homogeneous.

In this note we address the problem of genericity for singularities of flat 3-webs. Our considerations are local, all the objects involved are smooth or analytic.

\section{Genericity via transversality}

The 3-web, formed by integral curves of Eq. (1), is flat if and only if $K=$ $\left(K_{3}, K_{2}, K_{1}, K_{0}\right)$ satisfy a certain (rather involved) nonlinear PDE of second order. Following Blaschke [9], let us normalize one-forms, vanishing on the web leaves, to satisfy the condition $\sigma_{1}+\sigma_{2}+\sigma_{3}=0$. For example, one may choose

$$
\begin{aligned}
& \sigma_{1}=\left(p_{2}-p_{3}\right)\left(d y-p_{1} d x\right), \quad \sigma_{2}=\left(p_{3}-p_{1}\right)\left(d y-p_{2} d x\right), \\
& \sigma_{3}=\left(p_{1}-p_{2}\right)\left(d y-p_{3} d x\right),
\end{aligned}
$$

where $p_{1}, p_{2}, p_{3}$ are the roots of (1) at a non-singular point $(x, y)$. The above normalization defines the so-called Chern connection form $\gamma$ by $d \sigma_{i}=\gamma \wedge \sigma_{i}$. Its derivative $d \gamma$ is the Blaschke curvature. Thus the hexagonality of the web amounts to the equation $d \gamma=0$, which involves the second order derivatives of $p_{i}$. Manipulating elementary symmetric functions of $p_{i}$, one rewrites this equation in terms of $K$ and its derivatives up to the second order.

The study of generic properties of solutions to PDEs was mainly concentrated on equations of order one. These properties are relatively well understood in terms of Legendrian and Lagrangian singularities (see [8,19]).

Our approach is based on the Thom ideas on genericity via transversality and is close to those of $[12,15]$, though results obtained in these papers are not applicable here, as the PDE $d \gamma=0$ for $K$, being linear in 2nd derivatives, has vanishing coefficients of these derivatives. Thus the Cauchy-Kowalevskaya Theorem does not work and no existence theorem can help us to analyze the space of solutions.

Consider the set $\mathcal{H}$ of map germs $K:\left(\mathbb{R}^{2}, 0\right) \rightarrow \mathbb{R}^{4}$ which solve the equation $d \gamma=0$. We adopt here a naive and old-fashioned point of view on perturbations, namely, we call $\widetilde{K} \in \mathcal{H}$ a perturbation of $K \in \mathcal{H}$ if, at the base point, the values of $\widetilde{K}$ and its first derivatives are close to the corresponding values of $K$. (Note that our knowledge of the subspace of $\mathcal{H}$, describing singular hexagonal 3 -webs, is rather limited. For instance, it is not clear if higher derivatives of a perturbation $\widetilde{K}$ are close to those of $K$.) 
For each $\left(k_{3}, k_{2}, k_{1}, k_{0}\right) \in \mathbb{R}^{4} \backslash\{(0,0,0,0)\}$ there are solutions $K \in \mathcal{H}$ such that $K(0,0)=\left(k_{3}, k_{2}, k_{1}, k_{0}\right)$. In fact, a cubic form $g(p)=k_{3} p^{3}+k_{2} p^{2}+k_{1} p+k_{0}$ can be brought by a Möbius transform (i.e. by a transform that is fractional linear in $\mathrm{p}$ ) to one of the following forms: 1) $p^{3}$ (triple root), 2) $p^{2}$ (double root), 3) $p(p-1)(p+1)$ (distinct roots). Thus, applying a suitable local diffeomorphism, we get a desired solution from the normal form 2) of classification list (2), if $g(p)$ has a triple root; or from the normal form 3), if $g(p)$ has a double root; or from the non-singular flat 3-web. Therefore for any $K \in \mathcal{H}$ there is a small perturbation $\widetilde{K}$ such that $\widetilde{K}(0,0) \neq(0,0,0,0)$. This means that all web directions are well defined at each point. Choosing local coordinates so that none of the web leaves at the origin is tangent to the $y$-axis, one ensures $K_{3}(0,0) \neq 0$. Dividing (1) by $K_{3}$, one reduces our equation to a monic one:

$$
p^{3}+a(x, y) p^{2}+b(x, y) p+c(x, y)=0 .
$$

Finally, "killing" the coefficient by $p^{2}$ by a differential analog of Tschirnhausen transformation

$$
y=f(\tilde{x}, \tilde{y}), x=\tilde{x}, \text { with } 3 f_{\tilde{x}}+a(\tilde{x}, f)=0
$$

(see [1] for more detail), one arrives at the normal form

$$
F(x, y, p)=p^{3}+A(x, y) p+B(x, y)=0 .
$$

This equation gives a map germ $W:\left(\mathbb{R}^{2}, 0\right) \rightarrow \mathbb{R}^{2}$ by $W(x, y)=$ $(A(x, y), B(x, y))$. Now the Chern connection form in the chosen normalization of the forms $\sigma_{i}$ is

$$
\gamma=\frac{\left(2 A^{2} A x-4 A^{2} B y+6 A B A y+9 B B x\right)}{4 A^{3}+27 B^{2}} d x+\frac{\left(4 A^{2} A y+6 A B x+18 B B y-9 B A x\right)}{4 A^{3}+27 B^{2}} d y .
$$

Then the flatness of the web manifests itself as a nonlinear PDE of the second order $d \gamma=0$. (See [1] for more detail and the exact form of this PDE. For the ODE without the quadratic term (6), the explicit formula for the curvature first appeared in [22].)

Consider the variety $E_{2}$, defined by equation $d \gamma=0$, in the jet space $J^{2}\left(\mathbb{R}^{2}, \mathbb{R}^{2}\right)$. Total derivatives of this equation with respect to $x$ and $y$ give the prolonged manifold $E_{k}$ in each jet space $J^{k}\left(\mathbb{R}^{2}, \mathbb{R}^{2}\right)$ for any $k \geq 2$. Differentiating $W$ we obtain a (possibly singular) parameterized manifold $\Sigma_{k} \subset J^{k}\left(\mathbb{R}^{2}, \mathbb{R}^{2}\right)$ of dimension $\operatorname{dim} \Sigma_{k} \leq 2$.

Conversely, any map $W:(x, y) \rightarrow(A(x, y), B(x, y))$ with $\delta:=-4 A^{3}-$ $27 B^{2} \not \equiv 0$ locally determines a 3 -web, namely, the web of integral curves of the implicit ODE (6). Any property of the web can be described in terms of the map $W$ and its derivatives.

In particular, the failure of the following regularity condition on the criminant $C$

$$
\operatorname{rank}\left((x, y, p) \mapsto\left(F, F_{p}\right)\right)=2,
$$


used in [1,14], determines a submanifold $R$ of codimension 3 in $J^{1}\left(\mathbb{R}^{2}, \mathbb{R}^{2}\right)$ by:

$$
\left\{\begin{array}{l}
4 A^{3}+27 B^{2}=0 \\
A_{x} B_{y}-A_{y} B_{x}=0 \\
3 B A_{x}-2 A B_{x}=0 \\
3 B A_{y}-2 A B_{y}=0
\end{array}\right.
$$

One obtains equations (9) by direct computation, substituting the double root $p$ of (6), satisfying $F=F_{p}=0$, into the determinantal variety determined by the rank drop

$$
\operatorname{rank}\left((x, y, p) \mapsto\left(F, F_{p}\right)\right)<2 .
$$

Observe that the regularity condition (8) implies that $M$ and $C$ are smooth.

Let us call the set of points $m$ on the criminant $C$ a Legendrian locus, if the tangent plane to $M$ at $m \in C \subset M$ coincides with the contact plane $\mathrm{d} y-p \mathrm{~d} x=0$ at $m$. The Legendrian locus is determined by the following conditions:

$$
d F \wedge(\mathrm{d} y-p \mathrm{~d} x)=0, \quad F=F p=0 .
$$

Direct computation yields the following equations for the corresponding variety in $J^{1}\left(\mathbb{R}^{2}, \mathbb{R}^{2}\right)$, which we denote by $L$ :

$$
\left\{\begin{array}{l}
4 A^{3}+27 B^{2}=0 \\
6 A B A_{x}-9 B^{2} A_{y}+6 A B B_{y}-4 A^{2} B_{x}=0
\end{array}\right.
$$

Note that the variety $L$ is of codimension 2 .

Definition 2. We call a flat web, formed by integral curves of Eq. (6), generic if the parameterized manifold $\Sigma_{1}$ is transverse to the variety $R \cup L$.

Remark. The varieties $L$ and $R$ are defined in geometric terms. Therefore they are invariant with respect to any diffeomorphisms preserving the form of Eq. (6). A diffeomorphism preserves this form if it respects the condition $p_{1}+p_{2}+p_{3}=0$ and does not send any of the roots $p_{i}$ to infinity.

\section{Generic singularities of flat 3-webs}

Theorem 1. A generic singular flat 3-web germ defined by the implicit cubic ODE (6) is diffeomorphic either to the web formed by integral curves of the equation

$$
p^{3}+2 x p+y=0
$$

if all 3 web leaves are tangent at the base point, or to the web, formed by the lines $x=c s t$ and by the integral curves of the equation

$$
p^{2}-x=0
$$

if only 2 web leaves are tangent at the base point. 
Proof. Let us fix the base point $\left(x_{0}, y_{0}\right)$ of the web germ and the point $m=$ $\left(x_{0}, y_{0}, p_{0}\right) \in C \subset M$. Since the variety $R$ is of codimension 3 , the surface $\Sigma_{1}$ does not intersect $R$. Hence the regularity condition (8) is satisfied. The variety $L$ is of codimension 2 . Therefore the surface $\Sigma_{1}$ can meet $L$ only in isolated points.

Consider first the case of a triple root of (6). As shown in [1] (see Lemma 2 there), the contact plane is tangent to $M$ at $m$. (This amounts to $B_{x}=0$ at $\left(x_{0}, y_{0}\right)$, since one has $p_{0}=0$ for the normalization (6).) Thus $\Sigma_{1}$ intersects $L$ at a point. Due to genericity, this point is isolated and the criminant $C$ is transverse to the contact plane field in some punctured neighborhood of $m$. Consequently the conditions of Theorem 7 of [1] are satisfied and Eq. (6) is equivalent to the form (11) with respect to some local diffeomorphism at $\pi(m)=\left(x_{0}, y_{0}\right)$.

Now consider the case of double root. Equation (6) has a quadratic factor:

$$
p^{3}+A(x, y) p+B(x, y)=\left(p^{2}+a(x, y) p+b(x, y)\right)(p-a(x, y))=0 .
$$

At the point $\left(x_{0}, y_{0}\right)$ we have a simple root $p_{1}=a\left(x_{0}, y_{0}\right) \neq 0$ and a double root $p_{0}=-a\left(x_{0}, y_{0}\right) / 2$. Since these roots are distinct, the point $\left(x_{0}, y_{0}, p_{0}\right)$ lies on the surface defined by the quadratic equation $f(x, y, p)=p^{2}+a(x, y) p+b(x, y)=$ 0 . Hence the regularity condition (8) is now fulfilled for the function $f$. If $\Sigma_{1}$ misses the variety $L$, then the contact plane is not tangent to $C$ at $m$. Due to the regularity condition, Theorem 5 of [1] implies that our web is equivalent to the normal form (12). Note that for this form the discriminant $x=0$ is a leave of one of the web foliations. Moreover, the discriminant curve $a^{2}-4 b=0$ of $p^{2}+a(x, y) p+b(x, y)$ is smooth. In fact, the regularity condition implies that at least one of the expressions $b_{x}+p_{0} a_{x}=b_{x}-\frac{a}{2} a_{x}$ and $b_{y}+p_{0} a_{y}=b_{y}-\frac{a}{2} a_{y}$ does not vanish at $\left(x_{0}, y_{0}, p_{0}\right)$. Thus the gradient of $a^{2}-4 b$ is not zero.

Finally, if $\Sigma_{1}$ intersects $L$ at a point, then the point $m$ of the criminant is Lagrangian. As we have mentioned before, the discriminant curve is tangent to the vector field $\partial_{x}+a \partial_{y}$ at each point $(x, y) \neq\left(x_{0}, y_{0}\right)$, therefore this also holds true at $\left(x_{0}, y_{0}\right)$. But at the point $\left(x_{0}, y_{0}\right)$ the vector field $\partial_{x}-\frac{a}{2} \partial_{y}$ is tangent to the discriminant curve as well, since $m$ is Lagrangian. Thus $a\left(x_{0}, y_{0}\right)=-\frac{a\left(x_{0}, y_{0}\right)}{2}$ and the root is triple. This contradiction finishes the proof.

Remark 1. The web germ (12) is equivalent to the web germ of the web (11) at any point $\left(x_{0}, y_{0}\right) \neq(0,0)$ on the discriminant. See Fig. 1 . For more web portraits the reader may consult [24].

Remark 2. As examples of non-generic singular flat 3-webs, one easily constructs the following ones, starting from the well-folded singularity $p^{2}+a x^{2}=2 y$, $a=$ cst:

$$
(x p-2 y)\left(p^{2}+a x^{2}-2 y\right)=0, \quad\left(2 y p-x\left(2 y-a x^{2}\right)\right)\left(p^{2}+a x^{2}-2 y\right)=0 .
$$

(See [14] for the detailed study of well-folded singularities of implicit quadratic ODEs.) The point $(0,0,0)$ is Legendrian for the folded point $(0,0)$ of $p^{2}+a x^{2}=$ $2 y$, and all coefficients of the above cubic equations vanish at the base point. 

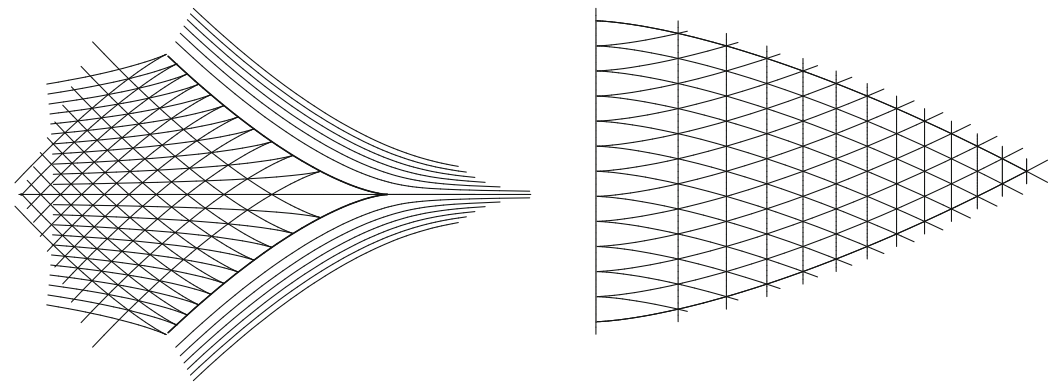

Fig. 1. Flat 3-webs of $p^{3}+2 x p+y=0$ (left) and of $p^{2}=x$ (right)

\section{Concluding remarks}

- Genericity and differential consequences of PDE It seems natural to expect a normal form with a triple root, where $\Sigma_{1}$ misses $L$. As it was shown in [1], the surface $\Sigma_{1}$ always meets $L$ whenever the regularity condition (8) holds true and all 3 roots $p_{i}$ of (6) coincide. It is interesting that this differential condition of order one follows from the PDE $d \gamma=0$, which is of order two.

- Perturbations of singular flat 3-webs The variety $L$ is of codimension 2 and the variety $R$ is of codimension 3, thus, due to Thom's Transversality Lemma, for a generic smooth map $U \subset \mathbb{R}^{2} \rightarrow \mathbb{R}^{2}$, its lift to $U \subset \mathbb{R}^{2} \rightarrow J^{1}\left(\mathbb{R}^{2}, \mathbb{R}^{2}\right)$ misses $R$ and has isolated points of intersection with $L$. But our map $W$ satisfies the PDE $d \gamma=0$ and its differential consequences, which defines an algebraic provariety of infinite codimension in the jet space $J^{\infty}\left(\mathbb{R}^{2}, \mathbb{R}^{2}\right)$. Therefore $W$ is not generic in the definition of Tougeron [27].

It remains a challenge to show rigorously that for any singular flat 3-web there is a small perturbation, within the class of flat 3-webs, that brings the web to a generic flat 3-web in the sense of Definition 2. For the case when $A, B$ are of order 2 the existence of such a perturbation can be shown directly, avoiding the difficult problem of existence of solutions to the singular PDE $d \gamma=0$. In fact, a change of variables $y=F(X, Y), x=G(X, Y)$ with $F(0,0)=G(0,0)=0$ preserving the form (6), sends (11) to the equation $P^{3}+\widetilde{A}(X, Y) P+\widetilde{B}(X, Y)=0$ with $\widetilde{A}_{X}(0,0)=2 G_{X}^{3}(0,0) / F_{Y}^{2}(0,0), \widetilde{A}_{Y}(0,0)=5 G_{X}^{2}(0,0) G_{Y}(0,0) / F_{Y}^{2}(0,0)$, $\widetilde{B}_{X}(0,0)=0$ and $\widetilde{B}_{Y}(0,0)=G_{X}^{3}(0,0) / F_{Y}^{2}(0,0)$. Therefore keeping $F_{Y} G_{X} \neq 0$, which ensures that $F, G$ are local coordinates, one can make all the first derivatives of $\widetilde{A}, \widetilde{B}$ arbitrarily small. Thus, according to our definition of perturbation in Section 2 , the web of $P^{3}+\widetilde{A}(X, Y) P+\widetilde{B}(X, Y)=0$ is the desired perturbation.

- Flat 3-webs of characteristics of PDEs In this note we define a singular web as a web of integral curves of some implicit ODE. This approach is motivated by examples from mathematical physics. For instance, any solution of the associativity equation

$$
u_{y y y}=u_{x x y}^{2}-u_{x x x} u_{x y y}
$$


determines the so called characteristic 3-web in $(x, y)$-plane by the following cubic implicit ODE:

$$
\mu^{3}+u_{x x x} \mu^{2}-2 u_{x x y} \mu+u_{x y y}=0, \text { where } \mu=-\frac{d x}{d y} .
$$

This web is flat. Each weighted homogeneous solution of the associativity Eq. (13) defines a structure of Frobenius 3-manifold (see [16]), for example, the web of normal form (11) is the characteristic web of a polynomial solutions to (13). (See $[4,5]$ for a geometric construction of such webs from Frobenius manifolds.)

Another class of examples of flat 3-webs comes as characteristic webs of integrable systems (see $[17,18]$ ) of hydrodynamic type:

$$
\vec{v}_{x}=\Lambda(\vec{v}) \vec{v}_{y},
$$

where $\vec{v}: U \subset \mathbb{R}^{2} \rightarrow \mathbb{R}^{3}$ is a vector function and $\Lambda(v)$ is a $3 \times 3$ matrix, whose entries depend explicitly only on $\vec{v}$. Again, each solution $\vec{v}(x, y)$ defines the characteristic web by the following implicit ODE

$$
\operatorname{det}[\Lambda(\vec{v}(x, y))+p \cdot \mathbf{1}]=0 .
$$

Here determinantal singularities may come into play and it would be surprising if generic singularities of characteristic webs of scalar PDE and those of systems of hydrodynamic types were the same. (Sometimes a system of hydrodynamic type is equivalent to a scalar PDE, see [6].)

Acknowledgements The author thanks J. Rieger for useful discussions. This research was partially supported by DAAD-CAPES Grant A/13/03350 and FAPESP Grant \#2014/178120 .

\section{References}

[1] Agafonov, S.I.: On implicit ODEs with hexagonal web of solutions. J. Geom. Anal. 19(3), 481-508 (2009)

[2] Agafonov, S.I.: On symmetries of singular implicit ODEs. Mat. Contemp. 43, 1-16 (2014)

[3] Agafonov, S.I.: Local classification of singular hexagonal 3-webs with holomorphic Chern connection and infinitesimal symmetries. Geom. Dedicata 176(1), 87-115 (2015)

[4] Agafonov, S.I.: Flat 3-webs via semi-simple Frobenius 3-manifolds. J. Geom. Phys. 62(2), 361-367 (2012)

[5] Agafonov, S.I.: Frobenius 3-folds via singular flat 3-webs. SIGMA Symmetry Integrability Geom. Methods Appl., 8 (2012), 078, 15 pp. arXiv:1206.0372

[6] Agafonov, S.I.: Linearly degenerate reducible systems of hydrodynamic type. J. Math. Anal. Appl. 222(1), 15-37 (1998)

[7] Arnold, V.I.: Geometrical Methods in the Theory of Ordinary Differential Equations, Grundlehren der Mathematischen Wissenschaften, vol. 250. Springer, New York (1983) 
[8] Arnold, V.I., Gusein-Zade, S.M., Varchenko, A.N.: Singularities of Differentiable Maps. Volume 1. Classification of Critical Points, Caustics and Wave Fronts. Birkhäuser, New York (2012)

[9] Blaschke, W.: Einführung in die Geometrie der Waben. Birkhäuser, Basel (1955)

[10] Blaschke, W., Bol, G.: Geometrie der Gewebe. Topologische Fragen der Differentialgeometrie. J. Springer, Berlin (1938)

[11] Cartan, E.: Les sous-groupes des groupes continus de transformations. Ann. Sci. École Norm. Sup 25(3), 57-194 (1908)

[12] Damon, J.: Generic properties of solutions to partial differential equations. Arch. Ration. Mech. Anal. 140(4), 353-403 (1997)

[13] Dara, L.: Singularités génériques des équations différentielles multiformes. Bol. Soc. Brasil. Mat. 6(2), 95-128 (1975)

[14] Davydov, A.A.: The normal form of a differential equation, that is not solved with respect to the derivative, in the neighborhood of its singular point. Funktsional. Anal. i Prilozhen. 19(2), 1-10 (1985)

[15] Dubois, J.-G., Dufour, J.-P.: Singularités de solutions d'équations aux dérivées partielles. J. Differ. Equ. 60(2), 174-200 (1985)

[16] Dubrovin, B.: Geometry of 2D topological field theories. Integrable systems and quantum groups, 120-348. Lecture Notes in Math. 1620. Springer, Berlin (1996)

[17] Ferapontov, E.V.: Systems of three differential equations of hydrodynamic type with a hexagonal 3-web of characteristics on solutions. Funct. Anal. Appl. 23(2), 151-153 (1989)

[18] Ferapontov, E.V.: Integration of weakly nonlinear semi-Hamiltonian systems of hydrodynamic type by the methods of web theory (Russian) Mat. Sb. 181(9)9, 1220-1235 (1990), translation in Math. USSR-Sb. 71(1), 65-79 (1992)

[19] Goryunov, V. V., Zakalyukin, V.M.: Lagrangian and Legendrian singularities. (English summary) Real and Complex Singularities, Trends Math., pp 169-185. Birkhuser, Basel (2007)

[20] Hénaut, A.: Planar web geometry through abelian relations and singularities. Inspired by S. S. Chern, 269-295, Nankai Tracts Math. 11. World Sci. Publ., Hackensack (2006)

[21] Mokhov, O.I., Ferapontov, E.V.: Associativity equations of two-dimensional topological field theory as integrable Hamiltonian nondiagonalizable systems of hydrodynamic type. Funct. Anal. Appl 30(3), 195-203 (1997)

[22] Mignard, G.: Rang et courbure des 3-tissus de C2. C. R. Acad. Sci. Paris Sér. I Math 329(7), 629-632 (1999)

[23] Nakai, I.: Webs and singularities. J. Singul. 9, 151-167 (2014)

[24] Nakai, I.: Web geometry of solutions of holonomic first order PDEs. Nat. Sci. Rep. Ochanomizu Univ. 53(1), 107-110 (2002)

[25] Nakai, I.: Web geometry of solutions of first order ODEs. Geometry and Foliations 2003, Kyoto, Invited talk. (http://gf2003.ms.u-tokyo.ac.jp/abstract/da)

[26] Thom, R.: Sur les équations différentielles multiformes et leurs intégrales singulières. Bol. Soc. Brasil. Mat. 3(1), 1-11 (1972)

[27] Tougeron, J.C.: Idéaux de fonctions différentiables, Ergebnisse der Math., vol. 71. Springer, New York (1972) 